\title{
Literaturwissenschaft und Kulturwissenschaften Eine Erwiderung
}

\author{
Von Gerhart v. Graevenitz (Konstanz)
}

Walter Haug beobachtet eine "periodisch" ausbrechende, an den Symptomen der "programmatischen Als-Metamorphose“ zu erkennende IchSchwäche des Faches. Die jüngste Variante heißt "Literaturwissenschaft als Kulturwissenschaft“. Warum, so fragt uns Walter Haug, darf oder kann die Literaturwissenschaft nicht sein, was sie ist, woher diese „Anfälligkeit gegenüber den Sicht- und Verfahrensweisen der Nachbardisziplinen“? Warum kann die Literaturwissenschaft nicht bei dem bleiben, um dessentwillen es sie gibt, beim "Sonderstatus der Literatur", der, so schlägt Walter Haug vor, sich zusammendrängen läßt in die „lebendig-gespannte() Konstanz des Dichterischen im Spannungsfeld seines Wirklichkeitszusammenhangs“. Der doppelte Tonus also muß stimmen und ist er stabil, dann kann zumal im Hinblick auf den „Wirklichkeitszusammenhang“ von Literatur eine wohldosierte Zusammenarbeit mit den Kulturwissenschaften durchaus förderlich sein. Das gilt zum Beispiel für Kulturwissenschaften vom Schlage der Clifford Geertzschen „interpretative cultural anthropology“, die sich der zentraleuropäischen Hermeneutik im Allgemeinen und den literaturwissenschaftlichen Kategorien im Besonderen anbequemt hat. Solche Selbstbegegnung im ethnologischen Fremden stärkt die Literaturwissenschaft. Man fragt sich freilich, warum diese Begegnung mit dem Exotischen noch nötig ist. Walter Haug hätte doch leicht darauf verweisen können, daß die Klassische Philologie und die Mediävistik ehrwürdige und ertragreiche kulturwissenschaftliche Traditionen besitzen. Aber ganz kann man sich dem Neuen eben nicht verschließen. Die alten Traditionen werden allenthalben mit Geertz, Turner, Clifford oder Greenblatt aufgefrischt. So lange das auf gemeinsamem „semiotischen“ Boden geschieht, mag es verträglich sein. Was aber darüber hinausgeht, nähert sich der perniziösen Als-Metamorphose, ist Selbstpreisgabe der Literaturwissenschaft. Unbegreiflich ist Walter Haugs düsteres Bulletin gewiß nicht. Wo immer häufiger und schneller von Sachkenntnis nur wenig belastete Empfehlungen zur medien- oder kulturwissenschaftlichen Ummöblierung der Literaturwissenschaften verbreitet werden, wo "Medien“ und „Kultur" sich wie ein Stichwörterausschlag über die literaturwissenschaftliche Massenproduktion legen, da kann man schon ab und zu verdrießlich oder pessimistisch werden. Aber Ärger und Melancholie sind schlechte Ratgeber. Walter Haugs Gegenvorschläge würden noch nicht einmal bei den Mißlichkeiten des Betriebs anschlagen, weil 
er die Symptome mit den Ursachen verwechselt. Denn so lästig der gedankenlose Gebrauch des „Kultur“-Fetisches sein mag, als Symptom verweist auch er auf ein grundsätzliches Problem, das die Verhältnisbestimmung von Literaturund Kulturwissenschaft nicht zu einer lästigen, sondern zu einer notwendigen Aufgabe macht. Walter Haugs auf die Innensicht ihrer Autarkie zurückverwiesene Literaturwissenschaft scheint nicht bemerken zu wollen, daß die Diskussion um die Kulturwissenschaften Artikulation einer allgemeinen Strukturveränderung der Wissenschaften ist. Diese auf ihrer Innensicht beharrende Literaturwissenschaft hat offenbar kein Interesse daran, sich an der Suche nach den Gründen für diese Strukturveränderungen zu beteiligen und ihren Platz in den sich verändernden Verhältnissen zu bestimmen. Schon was Walter Haug die "Literaturwissenschaft als Sozialgeschichte, als Psychoanalyse, als Ideologiegeschichte" nennt, und das Horrorkabinett ließe sich ja noch vergrößern, man denke nur an Strukturalismus und Rezeptionstheorie, das alles waren doch nicht einfach Schwächeanfälle des disziplinären Selbstbewußtseins. Sondern diese Paradigmen gehören, geliebt oder ungeliebt, zu jener „nachholenden Modernisierung “ 1 , die ausgangs der sechziger und in den siebziger Jahren nicht nur die Geisteswissenschaften, sondern den Wissenschaftsbetrieb wie die gesamte Kultur der Bundesrepublik dramatisch verändert hat. Denn das kann doch wohl nicht die Meinung Walter Haugs sein, daß wir die Germanistik der fünfziger und frühen sechziger Jahre als Identität festhalten, um unsere Literaturwissenschaft vor den Modernisierungsschüben in Sicherheit zu bringen, das hieße ja, Germanistik zu dem zu machen, was sie eine Zeitlang zu sein versuchte, zum Bollwerk des Antimodernismus. ${ }^{2}$ Einen dieser Modernisierungsschübe, den natürlich auch Walter Haug kennt, bezeichnet das Stichwort „neue Medien“. Er hat unsere Kultur verändert und wird sie weiter verändern. Nicht so, wie viele zunächst meinten, daß durch die neuen Medien Schrift, Buch und Text verdrängt würden. Aber die neuen Medien haben den kulturellen Kontext, die kulturellen Funktionen von Schrift, Buch und Text verändert. Darum kommen die Literaturwissenschaften, deren Gegenstände die Form von Schrift, Buch und Text haben, nicht umhin, sich mit dem Kontext ihres Mediums, mit den alternativen Medien auseinanderzusetzen. So ist zum Beispiel durch die apparative Bindung der "neuen Medien“ (einschließlich der "alten" Apparat-Medien Phonographie, Photographie und Film) der Medienbegriff insgesamt technischer und materieller geworden. Es genügt nicht mehr, mit der alten hermeneutischen Figur von „Buchstabe“ und

${ }^{1}$ Hartmut Böhme, Klaus R. Scherpe (Hrsg.), Literatur und Kulturwissenschaften. Positionen, Theorien, Modelle, rowohlts encyclopädie, Reinbek bei Hamburg 1996, 12.

2 Programmatisch in Emil Staigers Zürcher Rede. 
„Geist" und ihrem mehr oder minder weichen Platonismus aktuelle Medienanalyse zu betreiben. Weil aber die Basis des neuen Medienbegriffs die materielle Kultur der technischen Apparate ist und nicht der Buchstabe-GeistPlatonismus der alten Schrift-Begriffe, darum ziehen es viele Wissenschaftlerinnen und Wissenschaftler vor, das Ensemble der Kunst-, Medien- und Literaturwissenschaften näher an die von jeher mit der materiellen Kultur befaßten Wissenschaften heranzurücken, sie alle zusammen als „Kulturwissenschaften“ $\mathrm{zu}$ bezeichnen und in ihrem Kontext zu bestimmen, was unter aktuellen Voraussetzungen Schrift, Buch und Text bedeuten können. Dieser Begriff der „Kulturwissenschaften“ ist zunächst nur neutraler als der Begriff der „Geisteswissenschaften", hält anders als deren "Geist" und sein platonisch-hermeneutisches Schriftmodell die Probleme von Materialität, Medialität und Repräsentation für ganz verschiedene Konzeptualisierungen offen, auch für hermeneutische.

Das ist nur ein Grund dafür, daß sich der Begriff der „Kulturwissenschaften " beginnt, vor den der "Geisteswissenschaften" zu schieben. Aber schon dieser Grund genügt um deutlich zu machen, daß nicht der Begriff zählt, sondern die Wirklichkeitsveränderungen, die seine Semantik bestimmen. $\mathrm{Zu}$ dieser Semantik gehört, und das ist das Allgemeinste, was Walter Haugs Argumentation entgegenzuhalten ist, daß „Kulturwissenschaft“ einen veränderten Kontext aller mit Kultur befaßten Wissenschaften bedeutet, in dem Literaturwissenschaft sich nicht auflöst oder preisgibt, sondern auf den bezogen und nicht gegen ihn Literaturwissenschaft sich definiert. Walter Haugs Diagnose "Literaturwissenschaft als Kulturwissenschaft" richtet einen schiefen Blick auf das Problem, weil sie nur sehr unzureichend die Tatsache berücksichtigt, daß der Gegenbegriff von „Kulturwissenschaft“ der der „Geisteswissenschaft" ist und daß auch Geisteswissenschaft mit ihrem Unterbegriff "Literaturwissenschaft“ nicht einfach identisch ist. Umgekehrt gesagt, die von Walter Haug so ängstlich gehütete Spezifität der Literaturwissenschaft wird kein größeres oder kleineres Problem dadurch, daß sie kulturwissenschaftlich oder geisteswissenschaftlich definiert werden muß.

Die Semantik von „Kulturwissenschaft“ hält offenbar noch andere Fallgruben versteckt. Das läßt Walter Haugs Skizze der alten kulturwissenschaftlichen Debatte vermuten. Wie überall werden mehr rituell angeführt als gelesen die Neu-Kantianer Rickert und Windelband. Um ihrer Nennung als problematisches Beispiel einer kulturwissenschaftlichen Literaturinterpretation Adorno anschließen zu können, muß man freilich so ziemlich alles außer Betracht lassen, was zu Rickerts und Windelbands Zeiten „Kulturwissenschaft" hieß. Alle, die sich im 19. und 20. Jahrhundert um "Culturwissenschaft" bemühten, die Boeckh, Steinthal, Lazarus, Simmel, Weber oder Lamprecht, haben bei allen ihren sonstigen Unterschieden eines gemeinsam, nämlich das gegen die idealistische Geist-Metaphysik und Geschichtsphilosophie 
gerichtete historistische Erbe. Dieses historistische Erbe hat zum Beispiel Franz Boas an die amerikanische "cultural anthropology “ weitergereicht. ${ }^{3}$ Demgegenüber steht Adorno in eben der Tradition hegelianischer Geschichtsphilosophie, gegen die sich diese historische „Kulturwissenschaft“ gerichtet hat. Man kann ihr also nicht gut Adorno als ihr schlechtes Beispiel vorhalten. Auch Uwe C. Steiner weiß wohl in seiner in dieser Zeitschrift veröffentlichten Preisschrift nicht durchgehend, wovon er spricht, wenn er der von ihm gepriesenen „Kulturwissenschaft“ das zur Geschichtsphilosophie tendierende „Geist“-Paradigma entgegenhält und für letzteres Hegel und Dilthey gleichermaßen verantwortlich macht. ${ }^{4}$ In Bezug auf Geschichtsphilosophie zumindest ist das falsch, denn Diltheys "Geisteswissenschaften“ entsprangen nicht anders als die konkurrierenden Entwürfe von „Kulturwissenschaften“ der historistischen Kritik der Geschichtsphilosophie. Diese Gemeinsamkeit in der Abwehr der gescheiterten idealistischen Systeme bedingt, daß die älteren Begriffe von Geistes- und Kulturwissenschaften nicht als einfache Oppositionen zu konstruieren sind. Diltheys Lehrer Moritz Lazarus hat zusammen mit Heymann Steinthal ein Konzept von "Culturwissenschaft“ entworfen, dessen Gegenstand der „objektive Geist“ ist. Der Terminus klingt hegelianisch, meint aber einen Anti-Hegelianismus, nämlich keinen idealistischen, metaphysischen, sondern einen in den Objekten der materiellen Kultur manifesten Geist, ein an die medialen Strukturen der Sprache gebundenes Denken. Weshalb denn auch Georg Simmel, ein anderer Schüler von Lazarus und Steinthal, diese antihegelianische Halbherzigkeit umbenannte zur "objektiven Kultur" und sie, seinerseits einen anderen Hegelianismus begehend, als zunehmend unvermittelt der „subjektiven Kultur“ gegenüberstellt. ${ }^{5}$ Auch Dilthey benützt den Kultur- und den Geist-Begriff. Wo aber der ältere Begriff der Steinthal-Lazarusschen "Culturwissenschaft" besagt, daß Materialität und Struktur der Kultur die Physiognomie des Geistes prägen, da bestimmt Dilthey die „Kultur“ zum Materialbegriff, den „Geist“ zum Ziel- und Methodenbegriff der analytischen und synthetischen Durchdringung von „Kultur“6. Dilthey kann darum auch in seinem "Aufbau der geschichtlichen Welt in den Geisteswissenschaften“ den

\footnotetext{
${ }^{3}$ Vgl. Matti Bunzl, „Franz Boas and the Humboldtian Tradition: From Volksgeist and Nationalcharakter to an Anthropological Concept of Culture", in: George W. Stocking, Jr. (Hg.), ,Volksgeist' as Method and Ethic. Essays on Boasian Ethnography and the German Anthropological Tradition, The University of Wisconsin Press, Madison 1996, 17-78.

${ }^{4}$ Uwe C. Steiner, „Können die Kulturwissenschaften eine neue moralische Funktion beanspruchen?“, DVjs 71 (1997), 3-38; 7.

${ }^{5}$ Vgl. Klaus Christian Köhnke, „Der junge Simmel“, in: Theoriebeziehungen und soziale Bewegungen, Frankfurt a.M. 1996, 337-355.

${ }^{6}$ Vgl. Dietrich Harth, „Vom Fetisch bis zum Drama? Anmerkungen zur Renaissance der Kulturwissenschaften“, Anglia 114 (1996), 340-375; 348.
} 
Lazarusschen, kulturwissenschaftlich besetzten Begriff vom „objektiven Geist“ mühelos benützen. ${ }^{7}$

Es geht, sehr vereinfacht gesagt, nicht um den wechselseitigen Ausschluß von „Kultur" und „Geist“, sondern darum, ob der „Geist“ Subjekt oder Objekt von „Kultur“ ist. Die Kulturwissenschaft untersucht Materialität, Medialität, Strukturen und Geschichte von Kulturellem und Kulturen, um zu sehen, wie Geistiges produziert und konstruiert wird. Die Geisteswissenschaft macht Zeugnisse von Kultur und Kulturen zu Objekten, die als Erscheinungsweisen des Geistes zu deuten und zu verstehen sind. Die Kulturwissenschaft tendiert strukturell zum Pluralismus des Kulturellen, die Geisteswissenschaft zum Einheits- und Ganzheitsmodell des einen menschlichen Geistes. Das sieht Uwe C. Steiner ganz richtig: „Anders als der Begriff der Kultur war ,Geist“ von Hegel bis Dilthey und über ihn hinaus ein totalisierender, ein unifizierender Begriff." 8

Man sollte also die Parallelen zwischen alter und aktueller kulturwissenschaftlicher Debatte nicht überstrapazieren. Beide mögen entstanden sein oder neuen Auftrieb erhalten haben durch den Zusammenbruch philosophischer Großsysteme. Aber die älteren Kultur- und Geisteswissenschaften waren sich einig in der Frontstellung gegen die Geschichtsphilosophie, was Zonen erzeugt, in denen sie schwer auseinanderzuhalten sind. In der aktuellen Frontstellung von Kultur- und Geisteswissenschaften hat dieser historistische Impuls nur noch partielle Geltung. Sie ist viel stärker bestimmt durch die in der alten Debatte erst angelegte Opposition von Pluralität und Einheit als Letzthorizonten der Wissenschaft. Das ist es, was auch Walter Haug zumindest indirekt gegeneinanderführt: den kulturwissenschaftlichen Pluralismus des interdisziplinären, latent unseriösen „Spiels“ und die in Werk, Autonomie und Geschichte zentrierte hermeneutische Literaturwissenschaft. Wie bei Dilthey und den Diltheyanern wird eine vorzugsweise an der literarischen Hermeneutik exemplifizierte Geisteswissenschaft als Identitäts- und Einheitsentwurf gegen die moderne Zersplitterung der Wissensgebiete, ihre ungehemmte Vervielfältigung in den Kulturwissenschaften gehalten. Genau so aber ist Kulturwissenschaft aufzufassen: als der pluralistische Kontext für die Selbstreflexivität einer pluralistischen Literaturwissenschaft und nicht als der ungleiche Gegenpart in einer verfehlten Konfrontation.

\footnotetext{
${ }^{7}$ Vgl. Klaus Christian Köhnke, „Der junge Simmel“ (Anm. 5), 393.

${ }^{8}$ Uwe C. Steiner (Anm. 4), 7.
} 
II.

Die genauere Betrachtung des kulturwissenschaftlichen Pluralismus beginne ich mit einem historischen Rückblick.

Mit der Bezeichnung „Völkerpsychologie“ haben Heymann Steinthal und Moritz Lazarus ihrem Entwurf einer "Culturwissenschaft" Eingang eher ins Kuriositätenkabinett als ins Pantheon der Wissenschaftsgeschichte verschafft. Tatsächlich handelt es sich um einen frühen Versuch, die zunehmende disziplinäre Vereinzelung des modernen Wissens auf einer höheren Ebene wieder zusammenzuführen, ohne die Errungenschaften der Fächerkompetenzen preiszugeben. „Mit dem Drange der heutigen Wissenschaft nach Specialisierung der einzelnen Disciplinen hält das entgegengesetzte Bedürfniß nach Zusammenfassung und gegenseitiger Durchdringung derselben gleichen Schritt." 9 Alle, „welche die geschichtlichen Erscheinungen der Sprache, der Religion, der Kunst und Literatur und Wissenschaft, der Sitte und des Rechts, der gesellschaftlichen, häuslichen und staatlichen Verfassung ..., welche das geschichtliche Leben der Völker nach irgend einer seiner mannigfaltigen Seiten ... erforschen “10, sollen dies unter dem gemeinsamen Gesichtspunkt von „Psychologie“ tun. Die „mannigfaltigen Seiten“ unter einem Gesichtspunkt, perspektivierter Pluralismus also bildet die formale Struktur dieser Meta-Wissenschaft. Strukturell analog wird auch der junge Georg Simmel, ein Schüler dieser kulturwissenschaftlichen Völkerpsychologie, sein Verständnis von „Sociologie“ entwerfen: „Sie ist eine eklektische Wissenschaft, insofern die Produkte anderer Wissenschaften ihr Material bilden. Sie verfährt mit den Ergebnissen der Geschichtsforschung, der Anthropologie, der Statistik, der Psychologie wie mit Halbprodukten; sie wendet sich nicht unmittelbar an das primitive Material, das andere Wissenschaften bearbeiten, sondern als Wissenschaft sozusagen zweiter Potenz, schafft sie neue Synthesen aus dem, was für jene schon Synthese ist. In ihrem jetzigen Zustand gibt sie nur einen neuen Standpunkt für die Betrachtung bekannter Thatsachen. “11

Das mag ein wenig nach der Hierarchie von Hilfs- und Superwissenschaften klingen. Wichtiger ist der Gedanke, daß aus der Transdisziplinarität Wissenschaften „zweiter Potenz" entstehen, die im Falle der Soziologie sich wieder disziplinär konsolidieren, während sie im Falle der „Kulturwissenschaft“ die Bezeichnung für eben diese Transdisziplinarität geblieben ist. In dieser wissenschaftstheoretischen Tradition des Begriffs umschreiben z. B. Hartmut Böhme und Klaus R. Scherpe „Kulturwissenschaft“ heute als „Form der Modera-

\footnotetext{
${ }^{9}$ Zeitschrift für Völkerpsychologie und Sprachwissenschaft 2 (1862), [513].

${ }^{10}$ Zeitschrift für Völkerpsychologie und Sprachwissenschaft 1 (1861), 1.

11 Georg Simmel, „Über sociale Differenzierung“, in: Georg Simmel, Gesamtausgabe, II: Aufsätze 1887 bis 1890, Über sociale Differenzierung, Die Probleme der Geschichtsphilosophie (1892), hrsg. Heinz-Jürgen Dahme, Frankfurt a.M. 1989, 104-295; 116.
} 
tion“, als ein „Medium der Verständigung, eine Art Kunst der Multiperspektivität ..., um die heterogenen, hochspezialisierten, gegeneinander abgeschotteten Ergebnisse der Wissenschaften zu ,dialogisieren', auf strukturelle Gemeinsamkeiten hin transparent zu machen .... Kulturwissenschaft ist in diesem Verständnis nicht eine Einzelwissenschaft, sondern eine Metaebene der Reflexion und eine Form der beweglichen Verschaltung, vielleicht auch eine Steuerungsebene für die Modernisierung der Geisteswissenschaften. “12

Was oft nur wie eine Vertröstung auf konkretere Tage klingt, etwa die Umschreibung der „Kulturwissenschaften als Such- und Reflexionsbegriff“"13, das bezeichnet zunächst nur den strukturellen Sachverhalt, daß „Kulturwissenschaft" ein Kontextbegriff ist, der darum besser und korrekter als Plural „Kulturwissenschaften“ erscheinen sollte. Als plurale tantum immerhin mag auch der Singular durchgehen. Kulturwissenschaft ist kein Angriff auf die Identität der Literaturwissenschaft oder irgend einer anderen Disziplin, nur eben ein Kontext, der freilich die disziplinären Orientierungen in neue Dimensionen und Perspektiven rückt und ihnen neue Selbstreflexivität abverlangt. Es wäre umgekehrt eine hoffnungslose Überforderung der Literaturwissenschaft, wollte sie sich in irgendeiner ihrer Gestalten an die Stelle dieses Kontextes setzen.

Dieser strukturelle Begriff bleibt aber kein leerer Transzendentalismus. Er hat konkrete Auswirkungen in den Disziplinen, und das kann man leichter in der Ethnologie als in der Literaturwissenschaft studieren. In einer ihrer Vorformen, der „Völkerpsychologie“, hat die Ethnologie früh angefangen, Konzeptualisierungen des Pluralismus zu entwickeln. Das hat nicht zuletzt mit ihrem Gegenstand, der Vielfalt der Kulturen zu tun. Jedenfalls gehören zur Semantik von „Kulturwissenschaft“ zwei Ebenen, der allgemeine und strukturelle Pluralismus einerseits und die konkrete Konzeptualisierung von Pluralismus in disziplinären Kulturwissenschaften wie der Ethnologie andererseits. Die „Kunst der Multiperspektivität“ bezeichnet die allgemeine, ein „material gefüllter Begriff von Kulturwissenschaft“ die andere Ebene. ${ }^{14}$ Die Ethnologie ist für die Literaturwissenschaft nicht darum attraktiv geworden, weil Feldforschung auf dem Gebiet der Goetheschen Romanhelden getrieben werden soll, sondern weil der literaturwissenschaftliche Pluralismus sich als ein anderweitig, eben in der Ethnologie längst verarbeitetes Phänomen kultureller Pluralität wiederentdecken kann.

Was Georg Simmel „Eklektizismus“ nennt, was Pierre Bourdieu als „me-

12 Hartmut Böhme, Klaus R. Scherpe (Hrsg.), Literatur und Kulturwissenschaften (Anm. 1), 12.

${ }^{13}$ Georg Bollenbeck, „Die Kulturwissenschaften - mehr als ein modisches Label?", Merkur (1997), 259-265; 263.

${ }^{14}$ Hartmut Böhme, Klaus R. Scherpe (Hsgg.), Literatur und Kulturwissenschaften (Anm, 1), 12 f. 
thodologischen Polytheismus" 15 in Anspruch nimmt, das hat der in sehr kurzer Frist zum kulturwissenschaftlichen Kirchenvater avancierte Clifford Geertz zum Thema seiner methodologischen Reflexion gemacht, allerdings nicht der immer wieder mehr oder minder dicht beschriebene HahnenkampfGeertz, auch nicht der Geertz der durch gedankenlose Wiederholungen ziemlich entstellten „Kultur als Text"-Formel. Wichtiger ist hier der Geertz, der seine "interpretative cultural anthropology" begreift als reflexive Meta-Wissenschaft für die "multiple distinct anthropologies". In der Beschreibung von George E. Marcus und Michael M.J. Fischer befand sich diese "anthropology“ genannte Kulturwissenschaft 1986 im Zustand der „fragmentation and disunity“. "A period of experimentation is characterized by eclecticism, the play of ideas free of authoritative paradigma, critical and reflexive views of subject matter, openness to diverse influences embracing whatever seems to work in practise, and tolerance of uncertainty about a field's direction and of incompleteness of its projects. " 16

In diesem „experimentellen Zustand“17 bedeutete Geertz’ „anthropology“ nicht einfach, wie manchmal herablassend diesseits des Atlantik angemerkt wird, die verspätete Übernahme alteuropäischer Hermeneutik, sondern, vergleichbar der historiographischen Narrativik Hayden Whites oder Arthur C. Dantos, die von der angelsächsischen Sprachphilosophie inspirierte transdisziplinäre Reflexion auf die Bedingung der Möglichkeit disziplinärer Forschung: nicht umsonst stammt der Begriff der „dichten Beschreibung“ von dem Oxforder Sprachphilosophen und Erkenntnistheoretiker Gilbert Ryle. Geertz' disziplinäres Selbstverständnis ist eine methodologische Konzeptualisierung des allgemeinen kulturwissenschaftlichen Pluralismus. Geertz expliziert den Zeichen- und Handlungs-Begriff seines "semiotischen“ Kulturverständnisses unter wechselnden theoretischen Bezügen. Er beruft sich u.a. auf Talcott Parsons, Wittgenstein, Cassirer, Dilthey, Kenneth Brooke, Northrop Frye, Lévi-Strauss. „Kultur als eine Montage von Texten“ ist auf Max Weber bezogen und meint keinen literaturwissenschaftlichen Text-Begriff, sondern einen mit der "Gewebe“-Metapher belegten, auf Handlung und Sinn bezogenen soziologischen Struktur- und Komplexitätsbegriff. Kultur, bezogen auf das Komplexitätsproblem, wird als „Ensemble von Texten, die ihrerseits wieder Ensembles sind “18 bezeichnet, und um diese Komplexitätsstufungen angemessen analysieren und beschreiben zu können, ist ein „Ensemble“ para-

15 Pierre Bourdieu, Loic J.D. Wacquant, Reflexive Anthropologie, Frankfurt a.M. 1996, 54.

16 George E. Marcus, Michael M. J. Fischer, Antbropology as Cultural Critique. An Experimental Moment in the Human Sciences, Chicago, London 1986, S. VII, X, XII.

${ }_{17}$ Dietrich Harth (Anm. 6), 345.

18 Clifford Geertz, Dichte Beschreibung. Beiträge zum Verstehen kultureller Systeme, 4. Auflage, Frankfurt a.M. (1. Auflage 1987), 253, 259. 
digmatischer und disziplinärer Orientierungen, Bourdieus „methodologischer Polytheismus“ vonnöten. Dabei meint "Ensemble“ keinen anything-goes-Eklektizismus, nicht die von Walter Haug gefürchtete interdisziplinäre Flatterhaftigkeit, sondern den Eklektizismus vom Simmelschen Typ, den theoretisch wie praktisch perspektivierten Pluralismus. Die Minimalforderung an diesen perspektivierten Pluralismus ist, daß er einerseits in einem offenen Feld von theoretischen Paradigmen die Wahl seiner Orientierungen und Methoden nach den Erfordernissen des Forschungsinteresses und der Gegenstandsbeschaffenheit trifft, und daß er andererseits die Entscheidung für ein dominantes Paradigma, die konkrete Perspektivierung seiner vielfältigen methodologischen Kontexte in Bezug auf die praktischen Forschungserfordernisse theoretisch begründet. Das entspricht strukturell dem, was Karlheinz Stierle für einen aktuellen Begriff von Literaturwissenschaft formuliert hat: es wird möglich, „sich der zum Teil bedeutenden Einsichten der humanwissenschaftlichen Disziplinen Geschichte, Soziologie, Psychologie, Sprachwissenschaft und Anthropologie zu öffnen, ohne sich doch ihnen auszuliefern. Es ist kein billiger Eklektizismus, sondern wohlverstandene Interdisziplinarität (die immer einen disziplinären Fluchtpunkt haben muß, wenn sie nicht zur Extradisziplinarität werden soll), wenn die Literaturwissenschaft sich gemäß ihren eigenen Interessen aus den sie umgebenden Wissenschaften eine Koine herausarbeitet, die ihren eigenen Bedürfnissen entspricht. ${ }^{19}$

Als Vorbild für einen solchen perspektivierten Pluralismus führt Geertz Alfred Schütz an, den „Philosophen und Soziologen ..., dessen Werk den großangelegten ... Versuch darstellt, Ansätze von Scheler, Weber und Husserl mit solchen von James, Mead und Dewey zu verschmelzen “20. Geertz, der einen ähnlich breiten Paradigmen-Kontext für sich in Anspruch nimmt, nennt die Hermeneutik Paul Ricoeurs als Bezugspunkt seiner theoretischen Perspektivierung. Dabei ist die Position Ricoeurs nicht allein Bezugspunkt, sondern zugleich Modell einer solchen Perspektivierung. Denn mit der Berufung auf Ricoeur zentriert Geertz seine interpretative Methode in einer Hermeneutik, die eine Reformulierung von Verstehenstheorie unter der Voraussetzung pluraler Theorieparadigmen leistet. Bekanntlich stellt Ricoeurs Verstehenstheorie eine hermeneutische Perspektivierung strukturalistischer und sprachpragmatischer Theoreme dar. Geertz' Berufung auf Ricoeur erfültt also die Mindestanforderung an das methodologische Konzept des "perspektivierten Pluralismus": er zentriert seine offene und vielfältige paradigmatische Orientierung, und er zentriert mit Hilfe eines Modells, das selbst als theoretische Begründung des "perspektivierten Pluralismus“ zu lesen ist. Diese Grundstruktur der Ge-

19 Karlheinz Stierle, „Literaturwissenschaft", in: Ulf Ricklefs (Hrsg.), Fischer-Lexikon Literatur, 3 Bände, Fischer Taschenbuch 4566, Frankfurt a. M., II, 1156-1185, $1171 \mathrm{f}$.

${ }^{20}$ Clifford Geertz, Dichte Beschreibung (Anm. 18), $138 \mathrm{f}$. 
ertz'schen Position behält übrigens auch dann ihre exemplarische Bedeutung, wenn man sie nur als eine Variante unter anderen im erweiterten Kontext der interpretativen Kulturanthropologie betrachtet. ${ }^{21}$

Walter Haugs Geertz-Fazit - „Das ist der hermeneutische Zirkel und das Prinzip der Überholbarkeit auf Angelsächsisch“ (S. 82) - ist vielleicht ein wenig unterkomplex geraten. Gerade das ist es aber, was der literaturwissenschaftlichen Hermeneutik gut täte, in der Auseinandersetzung mit den Kulturwissenschaften nicht ihre eigene Unterkomplexität wiederzuerkennen, sondern neue Komplexität hinzuzugewinnen.

Mit einigem Grund könnte Walter Haug sich ungerecht behandelt fühlen. Sein Beitrag ist für ein Kolloquium entstanden, für die fest etablierte Veranstaltungsform, die der pluralistischen Struktur unserer Wissenschaften am weitesten entgegenkommt. Als Herausgeber dieser Zeitschrift praktiziert er einen fröhlichen, wenn auch nicht permissiven Pluralismus. Daß die Redeweise von der Literaturwissenschaft angesichts der Realitäten ein Trugschluß ist, weiß auch er. Warum sollte er allein verantwortlich gemacht werden für die weit verbreitete doppelte Moral im Umgang mit dem Pluralismus: Wir akzeptieren ihn als unabänderlichen Zustand moderner Wissenschaft und sehnen uns doch jeder nach der monistisch domestizierten Wahrheit. Die zweifellos unumstößliche Tatsache, daß Trans- und Interdisziplinarität nur auf der Grundlage von Fachkompetenz sinnvoll sein kann, dient auch als schnelle Rechtfertigung solcher Sehnsüchte. Man kann sich dieses Verhalten in entfernter Analogie zur amerikanischen Demokratie vorstellen: eine pluralistische Gesellschaft, in der an Theorien, Methoden und Gegenständen so ziemlich alles möglich ist, repräsentiert sich in nur zwei Parteien, den Traditionalisten und den Modernisten, und ein starker Präsident, vorzugsweise der Inhaber der eigenen wissenschaftlichen Praxis, bestimmt, was Wahrheit und Inbegriff der Literaturwissenschaft ist. Um aber auf Walter Haugs Text zurückzukommen: Die Kontextualität von Literaturwissenschaft und Kulturwissenschaften läßt sich konkretisieren. Am einfachsten dadurch, $\mathrm{da} \beta$ ich andeute, wie der kulturwissenschaftliche Kontext von Walter Haugs literaturwissenschaftlichen Kernbegriffen aussieht.

III.

In Gestalt dreier „Dilemmata“ hat Walter Haug den Identitätskern seiner Literaturwissenschaft formuliert, die Elemente einer historistisch-hermeneutischen Philologie verbindet mit Elementen der idealistischen Ästhetik. Die

${ }^{21}$ Die beiden letzten Abschnitte entsprechen Ausführungen in G.v. G. und Gottfried Seebaß, „Literatur und Anthropologie. Forschungsprogramm des Sonderforschungsbe-

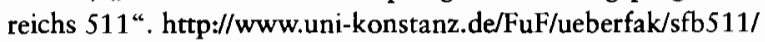


binäre Struktur der drei Dilemmata, die selbst wieder ein noch zu besprechendes Problem darstellt, möchte ich vermeiden. Ich nenne nur die vier Kernthemen, um die Walter Haugs Literaturwissenschaft zentriert ist, um sie im Folgenden einzeln und unterschiedlich ausführlich zu kommentieren:

- Individualität des Werks

- Autonomie des Werks

- Hermeneutik

- Geschichte

\section{Individualität des Werks}

Die medienhistorische Forschung hat gezeigt, wieviel der neuzeitliche WerkBegriff, angefangen beim neu erfundenen Titel-Blatt, den Individualisierungsverfahren des frühen Buchdrucks verdankt. Aus der Literaturgeschichtsschreibung wissen wir, wieviel theologische Vorstellungen von der geoffenbarten Schrift in diesen Werk-Begriff und seine Aura eingeflossen sind. Von der Sozialgeschichtsschreibung und Diskursanalyse wissen wir, welchen Einfluß politische, ökonomische, öffentliche und halböffentliche Institutionen auf die Durchsetzung dieses Werk-Begriffs beim gebildeten Publikum hatten. Die philosophische Ästhetik hat den Werk-Begriff auf ihre Kategorien von Autonomie und Identität hin bestimmt. Die Intertextualitätsdebatte hat uns die Augen dafür geöffnet, daß die ,Vielstimmigkeit‘ dem Singular dieser Identität Grenzen setzt. Die neuen Möglichkeiten interaktiver Textkonstitution haben die Grenzen des Werk-Begriffs noch durchlässiger gemacht. Wolf Kittler ${ }^{22}$ hat im 65 . Jahrgang dieser Zeitschrift aufgezeigt, welche Konsequenzen die Medientechniken für den Werk-Begriff der Editionswissenschaften haben können.

Das alles kann man beiseite rücken, und ich vermute, daß Walter Haug das alles beiseite läßt, wenn er ziemlich umstandslos einen idealistischen WerkBegriff und einen Fiktionsbegriff des 20. Jahrhunderts auf den arthurischen Roman anwendet. Das alles kann man auch zum Hintergrund machen für einen modernen Werk-Begriff, zu dessen Positivität gerade das „Risiko der Selbstgefährdung “23 gehört. Das alles kann man schließlich heranziehen, um positive wie negative Werk-Begriffe aufzufassen als produktions- wie rezeptionsästhetische Steuerungsversuche zur Durchsetzung bestimmter LiteraturKonzepte. Aber kann die Vielzahl solcher Aspekte des „Werk“-Begriffs anders denn „kulturwissenschaftlich“ genannt werden? Eines jedenfalls scheint mir sicher zu sein, daß ein literaturwissenschaftlicher Werk-Begriff, der mit den

22 Wolf Kittler, „Literatur, Edition und Reprographie“, DVjs 65 (1991), 206-235.

${ }^{23}$ Karlheinz Stierle, Ästhetische Rationalität. Kunstwerk und Werkbegriff, München 1997, 17. 
Adjektiven „individuell“ und „autonom“ auskommt, allenfalls noch indexikalische Verbindung mit dem Problem „Werk“ besitzt. Analytische Kraft erhält er erst wieder, wenn er sich auf seinen ganzen kulturhistorischen Kontext hin öffnet.

\section{Autonomie des Werks}

Ungern halte ich Walter Haug den von ihm perhorreszierten Adorno entgegen. Wenn „Autonomie“ aber mehr sein soll als ein Schlagwort, dann wird man das Reflexionsniveau der Asthetischen Theorie zumindest als Norm im Auge behalten müssen. „Ihr [der Kunst] gesellschaftliches Wesen bedarf der Doppelreflexion auf ihr Fürsichsein und auf ihre Relationen zur Gesellschaft. " ${ }^{24}$ Autonomie ist mit Negativität unlösbar verbunden, Autonomie definiert sich auf komplexe Weise über das, was sie nicht ist. Autonomie als Figur, als Erscheinung des Ästhetischen entsteht in selbstreflexiver Negation dessen, was sie an die ökonomische, gesellschaftliche und kulturelle Halbautonomie ihrer Entstehens- und Wirkungsbedingungen bindet. Was Walter Haug als Adornos Gesellschaftsallegorese verdächtigt, ist in ihren gelungenen Beispielen die Rekonstruktion des Ästhetischen als Heraustreten autonomer ästhetischer Reflexivität aus der kulturellen Bedingtheit in die Anschaulichkeit des Werks. Insofern kann gar keine Rede davon sein, daß die kulturwissenschaftliche Orientierung von Werkanalysen notwendig das Ästhetische in Kulturfahrpläne übersetzen muß und der kulturwissenschaftliche „Ansatz“ das Ende der „Kunst“ sei wie zuvor das sozialhistorische Rückübersetzen. Wenn sie das Ästhetische nicht selbst zum Thema macht, bedeutet jede Methode, angefangen beim grammatischen und rhetorischen Kommentar der Humanisten, das Ende des Ästhetischen. Nur wenn sie aus ihren eigenen Voraussetzungen zu erklären vermag, was das Ästhetische in ihrem Blick ist, findet eine Methode zu einem ihr eigenen Begriff des Ästhetischen. Umgekehrt ist die Berufung auf den tradierten Begriff des Ästhetischen aus der idealistischen oder hegelianischen Philosophie keine Garantie dafür, die Probleme von Autonomie und Reflexivität von Kunst abschließend geklärt zu haben. Dafür geben Adorno und Haug, wo sie sich ziemlich ähnlich sind, deutliche Beispiele. Beide nämlich siedeln die autonome Reflexivität der Kunst, die wie die Doppelstellung des Autonomen Reflexivität im Verhältnis zum Außen und zugleich Selbstreflexivität ist, beide siedeln diese Reflexivität ausschließlich in jenen "Werken“ der Hohen Kunst an, die den emphatischen Werkbegriff erfüllen und zugleich als Kanon des einigermaßen „Ewigen“ in Serie gehen. Zwei

24 Theodor W. Adorno, Asthetische Theorie, Gesammelte Schriften, VII, Frankfurt a.M. 1970, 337. 
Gegenbegriffe zieht diese Auffassung traditionellerweise nach sich, das "Wilde“ und das „Triviale“. Dem naiven „Mythos“ ist der reflexive Logos fremd und der trivialen Massenware widerspricht der Gedanke der Autonomie. Das Wilde, Mythische und die Waren sind nicht Geist vom Geiste der Autonomie, weder bei Adorno noch bei Haug.

Sollte sich Walter Haugs selbstverständliche Annahme, der balinesische Hahnenkampf könne nicht selbstreflexiv sein, intensiven Hahnenkampfstudien verdanken, so kann ich dem nichts Vergleichbares entgegen halten. Vorerst aber vermute ich, daß Walter Haug den Hahnenkampf gewissermaßen in nachidealistischer Routine und wie Uwe C. Steiner in der schon erwähnten Preisschrift den Mythos behandelt als „feste Kopplung von Kommunikationssequenzen ..., die der Möglichkeit ihrer Selbstreflexion ermangelt"25. Man darf daran erinnern, daß es ausschließlich eine Frage des wissenschaftlichen Paradigmas ist, ob man im Mythischen und Ethnischen Selbstreflexivität entdeckt oder nicht. Jedenfalls war der Lévi-Strauss'sche Strukturalismus eine methodische Möglichkeit, selbstreflexive Strukturen im mythischen Material aufzuzeigen. Man mag dieses Paradigma nicht mögen und mit guten Gründen ablehnen. Aber man kann schwerlich abstreiten, daß das Einlassen auf diejenigen Wissenschaften, die mit fremden und vergangenen Kulturen befaßt sind, daß also eine kulturwissenschaftliche Perspektive nötig ist, um angemessen diskutieren zu können, ob Selbstreflexivität in der Sache oder in der Betrachtungsweise begründet ist. Die normative Ausschließung der Möglichkeit von Selbstreflexivität im „Wilden“, „Mythischen“ oder „Populären“ jedenfalls ist dogmatisch. Immerhin muß man außerhalb der Germanistik heute mit der Möglichkeit rechnen, daß der balinesische Absolvent eines ethnologischen Studiengangs den Ausschluß des Hahnenkampfs aus dem Reich der Selbstreflexivität für ein ethnologisch interessantes Merkmal zentraleuropäischer Universitäten hält.

Adornos Ästhetische Theorie führt für die Kunst der Moderne aus, wofür Schillers Bürger-Rezension die Weimarer Skizze war, die Abwehr des falschen Bewußtseins im Nur-Populären. Was heute „Post-Moderne“ genannt wird, ist davon unter anderem unterschieden durch die Öffnung des Begriffs vom „Ästhetischen“ für populäre Massen- oder Warenkunst. Ich zitiere aus Albrecht Wellmers Zur Dialektik von Moderne und Postmoderne.

Benjamin sieht in der technisierten Massenkunst Elemente eines Gegengifts gegen die psychische Zerstörung der Menschen durch die industrielle Gesellschaft; während Adorno sie vor allem als Medien der Anpassung und der psychischen Manipulation begreift. Interessant ist nur die Antithese als solche: ich denke, daß Benjamins Analyse zumindest Potentiale der modernen Massenkunst andeutet - vom Film bis zur Rockmusik - die Adorno, aus Traditionalismus wie aus theoretischer Voreingenommenheit,

25 Uwe C. Steiner (Anm. 4), S. 36. 
nicht hat sehen können. Die Rockmusik als ,industrielle Volksmusik' wäre ein Testfall; ich denke, daß in der Rockmusik und in den Einstellungen, Wahrnehmungsweisen und Fertigkeiten, die sich im Zusammenhang mit ihr herausgebildet haben, ebensoviele Potentiale einer Demokratisierung und Entfesselung der ästhetischen Phantasie stecken wie solche einer kulturellen Regression. Solche Ambivalenzen gälte es, wie im Falle des Jazz, gegen Adorno zu verteidigen. ${ }^{26}$

Anführen läßt sich auch die soziologische Beschäftigung mit der Massenkultur. Im Triumvirat der sogenannten „reflexiven Modernisierung“, gebildet von Ulrich Beck, Anthony Giddens und Scott Lash, befaßt sich Letzterer vor allem mit der „ästhetischen Reflexivität der Kulturformen " ${ }^{\text {27 }}$ und zwar gerade auch der Formen ästhetischer Reflexivität, die die Kulturindustrie hervorbringt. Mag sein, daß auch diese Untersuchungen zuletzt nicht um Figuren von Autonomie, Negativität und Doppelreflexivität herumkommen. Der feine, aber wichtige Unterschied ist dann freilich, daß verschiedene Möglichkeiten ästhetischer Reflexivität als Teil und Instrument einer allgemeineren kulturellen Reflexivität neu bestimmt worden sind und Autonomie und Selbstreflexivität nicht mißverstanden werden können als automatisierte Absicherungsformeln tradierter Vorstellungen von Hoher Kunst. Das übrigens ist eine der Absichten des als „postmodern“ angeklagten Stephen Greenblatt, nicht Shakespeares ästhetische Autonomie und Selbstreflexivität in Zweifel zu ziehen, sondern sie zurückzuversetzen in den Kontext einer allgemeinen, sehr vielfältigen kulturellen Reflexivität und die Besonderheit des Ästhetischen gerade in diesem Kontext zur Anschauung zu bringen.

Ist Literaturwissenschaft wirklich nur dann Literaturwissenschaft, wenn sie die Definitionskompetenz für das „Autonome“ und das „Selbstreflexive“ denjenigen überläßt, die sich als Ethnologen mit nicht-westlichen Kulturen, als Philosophen und Soziologen mit den Veränderungen der modernen westlichen Kulturen befassen? Ich hielte es für keine schlechte Idee, wenn die Literaturwissenschaften versuchten, in dieser kulturwissenschaftlichen Diskussion mitzuhalten.

\section{Hermeneutik}

Weder ist Geertz einfach nur literaturwissenschaftliche Hermeneutik auf angelsächsisch, noch ist er das letzte Wort zur ethnologischen Hermeneutik. Die writing-culture-Debatte hat sich nicht nur mit Hermeneutik, sondern auch

\footnotetext{
${ }^{26}$ Albrecht Wellmer, Zur Dialektik von Moderne und Postmoderne. Vernunftkritik nach Adorno, Frankfurt a. M. 1985, 42.

27 Ulrich Beck, Anthony Giddens, Scott Lash, Reflexive Modernisierung. Eine Kontroverse, Frankfurt a. M. 1996, 241. Vgl. Scott Lash, „Reflexive Modernization: The Aesthetic Dimension“, Theory, Culture and Society 10/1 (1993), 1-24.
} 
mit Hermeneutik-Kritik beschäftigt. Autoren wie Dennis Tedlock, George E. Marcus, Dick Cushman, James Clifford - die Liste ließe sich verlängern haben das Diskursivitätsproblem und das Erkenntnisproblem von Anthropologie und Ethnologie verstärkt unter den Vorzeichen poststrukturalistischer Differenz- und Diskurstheorien behandelt. Marc Manganaro spricht von „moderner“ und „postmoderner“ Ethnologie. Letztere kritisiert unter der Prämisse einer Krise der Repräsentation die von den älteren „modernen“ Ethnologen beschriebene und praktizierte Textualität von Kultur. Man kann also ohne Gefahr zu großer Verallgemeinerungen sagen, die „interpretative cultural anthropology“ habe dieselben Paradigmenwechsel und -überlagerungen durchlaufen wie die Literaturwissenschaft. „Reading anthropology discursively would be impossible if not for the emergence of phenomenology, semiotics, structuralism, and the deconstructionist movement. "28 Selbstverständlich gibt es, wie in der Literaturwissenschaft auch, die Einwände gegen den Repräsentationsskeptizismus. Pierre Bourdieu diagnostiziert bei James Clifford und George E. Marcus einen „kaum verhüllten nihilistischen Relativismus“29. Auch vor dem Hintergrund der nachhermeneutischen Theorien behalten die ethnologischen Hermeneutik-Vorlieben ihre Gültigkeit. Gadamers "Gespräch“ ist als Utopieformel für Interkulturalität genauso beliebt wie Bachtins weniger hermeneutische „Dialogizität". Hier müßte auch in der Ethnologie eine Enttrivialisierung der Hermeneutik ansetzen. Dazu würde auch gehören, dem verbreiteten Irrtum zu begegnen, kulturwissenschaftliches Arbeiten sei per se gegen die Dekonstruktion gerichtet. ${ }^{30}$ Vielmehr gehört die Dekonstruktion zu den wichtigen Voraussetzungen neuer kulturwissenschaftlicher Forschung: ohne die differenztheoretische Schulung durch die Dekonstruktion ist die aktuelle Interkulturalitätsdebatte gar nicht zu führen. Man kann sich also ersparen, die literaturwissenschaftlichen Theoriedebatten um Hermeneutik oder Nicht-Hermeneutik unter ethnologischen Vorzeichen zu wiederholen. Man braucht vielmehr diese Debatten als Voraussetzungen dafür, kulturwissenschaftliche Fragen zu stellen. Eine solche Frage an die Hermeneutik lautet zum Beispiel, ob sie nicht eine der maßgeblichen Kulturtechniken zur „Aneignung des Fremden“ (S. 71) war. Walter Haug benützt diese Formel ganz unbefangen für das Verstehen historisch fremd gewordener Texte. Aber muß ich den Mediävisten daran erinnern, daß dieses verharmloste Verständnis der „Aneignung des Fremden“ einen Späthorizont der akademisierten Hermeneu-

${ }^{28}$ Marc Manganaro, „Textual Play, Power, and Cultural Critique. An Orientation to Modernist Anthropology“, in: ders. (Hg.), Modernist Anthropology. From Fieldwork to Text, Princeton 1990, 3-47; 16

29 Pierre Bourdieu, Reflexive Anthropologie (Anm. 15), 103.

${ }^{30} \mathrm{Vgl}$. das ansonsten verdienstvolle, von Doris Bachmann-Medick herausgegebene Buch: Kultur als Text. Die anthropologische Wende in der Literaturwissenschaft, Frankfurt a.M. 1996, 44. 
tik repräsentiert? Zur Blütezeit der typologischen Exegese ${ }^{31}$ in Patristik und Mittelalter bedeutete die auslegende Aneignung des Fremden zugleich ein Noch-Fremder-Machen des nicht Anzueignenden: das „Alte Testament“ war die figura des „Neuen“ und gerade nicht das Heilige Buch der Juden. Die typologische Aneignung und ihre aufwendige Theoretisierung waren begleitet von kriegerischen, macht- und ordnungspolitischen Aneignungen oder Aussonderungen des Fremden. Natürlich ist das alles bekannt. Aber man kommt doch in Versuchung zu fragen, ob ebenso bekannt ist, daß man die kulturwissenschaftliche Auseinandersetzung mit allen Aspekten der „Aneignung des Fremden" nicht einfach abdrängen kann in das der Germanistik eher befremdliche Gebiet der „post-colonial-studies“, daß vielmehr das Mittelalter auch den Germanisten ideale Anschauungsgegenstände böte, an ihnen die Konstruktionspraktiken und theorien der westlichen Expansionskultur zu studieren. Und das wäre keine Fragestellung jenseits der Literatur, auch keine Fragestellung, die von vornherein einen Sonderstatus von Literatur ausschlösse. Sollte die unerschöpfliche Phantasie der arthurischen Romane für die Inszenierung des Fremden, sollten die Orientalen, Schwarzen, Heiden, Riesen und Zwerge in ihrem Personal, die Lust am „surrealen Panoptikum“32, die Freude an strukturellen Synkretismen, sollten diese literarischen Modelle des Fremden so ohne jeden Bezug sein zu den Fremdheitsvorstellungen eines in Kreuzzügen erfahrenen Ritterpublikums, zur kirchlichen Definitionsmacht des „Fremden“ und zur politisch-ständischen Ideologie des „Eigenen“? Vielleicht zeigt diese Frage nur, daß ich vom arthurischen Roman so wenig verstehe wie vom balinesischen Hahnenkampf. Davon ist aber nicht die Tatsache berührt, daß Mediävisten zu einem der Hauptgebiete der neuen Kulturwissenschaften, der Interkulturalitätsforschung, zentrale Beiträge liefern könnten und daß es ihnen, sofern es diese Beiträge schon gibt, ${ }^{33}$ noch nicht hinreichend gelungen ist, sie auch als Beiträge zur allgemeinen kulturwissenschaftlichen Debatte ins Gespräch zu bringen. Die Rückkehr der Literaturwissenschaft in den Kontext der Kulturwissenschaften kann ja die Rückkehr der Mediävistik an die vorder-

${ }^{31}$ In einem denkwürdigen Disput über die Frage, ob die Doppelwegstruktur des arthurischen Romans mit dem figuralen Schema der typologischen Exegese in genetischem Zusammenhang stehe, hat Walter Haug Rainer Warning entgegengehalten: „Das würde voraussetzen, daß es im Mittelalter ein allgemeines figurales Dekmuster gab“ (Walter Haug, Literaturtheorie im deutschen Mittelalter. Von den Anfängen bis zum Ende des 13. Jahrbunderts. Eine Einführung, 1. Auflage, Darmstadt 1985, 98). Ich kann das nur so verstehen, daß für die literaturwissenschaftliche Behandlung eines zentralen Strukturelements des arthurischen Romans ein "kulturwissenschaftliches Argument ${ }^{\text {“ }}$ (allgemeines ... Denkmuster) eine wichtige Rolle spielt.

${ }^{32}$ Walter Haug, Literaturtheorie im deutschen Mittelalter (Anm. 31), 256.

${ }^{33}$ Es gibt sie natürlich. Ein jüngstes, noch unveröffentlichtes Beispiel ist die Dissertation von Waltraud Gut, Schwarz auf Weiß: Maske und Schrift des heraldischen Ornaments (MS 1998). 
ste Linie der Theoriebildung mit einschließen. Jedenfalls ist der hermeneutische „Tigersprung“, von dem Walter Haug spricht, kein Einwand gegen kulturwissenschaftliches Arbeiten, sondern einer seiner vornehmsten Gegenstände.

\section{IV.}

Unter Walter Haugs Begriffen der wichtigste ist der der Geschichte. Die im Sonderstatus der Literatur sich ereignende Selbstreflexion „heißt unweigerlich auch Bewußtsein der Geschichte" (S. 86). Kulturwissenschaft, so gesteht Walter Haug zu, verändert unser Geschichtsdenken und diese Veränderung sollte der Literaturhistoriker zum Anlaß nehmen, „sein Geschichtsdenken umzuakzentuieren“ (S. 86). Man könnte sich natürlich auch etwas weitergehende Befragungen der Kategorie „Geschichte“ vorstellen. Die Ethnologen debattieren derzeit eifrig, wie sie die methodischen Vorzüge der Historiographie wieder in ihre Arbeit integrieren können. Früher gaben sie sich kritischer. Johannes Fabian hatte zum Beispiel 1983 in Time and the Other ${ }^{34}$ gezeigt, wo das historische Denken mit seiner Verweigerung von Gleichzeitigkeit eine Methode zur Grenzziehung zwischen dem Eigenen und dem Fremden ist. Das wäre ein kulturwissenschaftlicher Kontext, in dem die Entautomatisierung der Vorstellungen von "Geschichte" auch in der Literaturwissenschaft diskutiert werden könnte, sicher mit weniger ideologiekritischen Begleitgeräuschen als in den Achtziger Jahren. Alles was heute unter „Historischer Anthropologie“ firmiert und sich schnell auf mehr oder minder gründlich durchdachte Kompromißformeln zwischen evolutionärer Natur und historischer Kultur zurückzieht, müßte sich an solchen radikaleren Kritiken der anthropologischen "Zeit"Prinzipien messen lassen.

Aber man soll keinen Dreisprung verlangen, wo schon der kleinste Schritt weg vom Pfad der historistischen "Geschichte“ so große Mühe bereitet. Auch die von Walter Haug akzeptierte neue kulturwissenschaftliche Lizenz zum „Einzelnen“, der systemischen oder philosophischen Regulierung Entzogenen, bleibt ja im Rahmen des alten Historismus. Die „Abwendung von globalen Modellen“, das Zurücktreten von "Gesetzlichkeiten“, also alles das, was "Geisteswissenschaft" oder "Culturwissenschaft" seinerzeit gemeinsam gegen die Geschichtsphilosophie vortrugen, ist wieder à l'ordre du jour. Schnell und konsequent schwenkt Walter Haug auf eine der systematischen Folgen ein, die der Historismus in seiner geisteswissenschaftlichen Spielart, genauer in seiner Diltheyschen Spielart, schon einmal hatte. Auch Walter Haug steuert auf den ,latenten Ahistorismus des historischen Sinns' zu, den Odo Marquard bei Dilthey aufgespürt hat und den Walter Haug formuliert als die dem „Inkon-

34 Johannes Fabian, Time and the Other. How Anthropology Makes its Object New York 1983. 
stanten", "Gegenläufigen“ abgerungene „Konstanz eines literarischen Konzepts“, die „lebendig-gespannte Konstanz() des Dichterischen im Spannungsfeld seines Wirklichkeitszusammenhangs“ (S. 86f.). „Konstanz“ - am Bodensee sieht man sich veranlaßt, die gemeinten anthropologischen Konstanten etwas unumwundener beim Namen zu nennen - Konstanten sind Konstrukte, die in ihrer historischen Erscheinungsweise dem Historischen zugleich wieder entzogen werden. Sie sind ein bißchen nach Art der negativen Theologie konstruierte Formen des Seins, die niemand sieht, es sei denn eingewickelt in den Gestalten des Seienden. Odo Marquard hat gezeigt, wie Dilthey mit solchen anthropologischen Konstrukten eine Art naturphilosophischen Schutzwall errichtet gegen den Rückfall der Geisteswissenschaften in die Geschichtsphilosophie. ${ }^{35}$ Er hat auch gezeigt, wie Dilthey versucht, den Gedanken von einer Natur des menschlichen Geistes zu verbinden mit dem Pluralitätsgedanken: der eine Geist kennt mehrere nicht hintergehbare Typen des Denkens. Die philosophische Anthropologie der Dilthey-Nachfolge hat aber diesen RestPluralismus noch weiter reduziert. In Erich Rothackers „Kulturanthropologie der Kulturstile“ bleibt zuletzt die Einheit eines Paradoxes. Der Diltheysche Dualismus von Geist und Natur, Verstehen und Erkennen wird zur anthropologischen Konstante des polaren Denkens. Rothacker geht es um „a priori einsichtige Gesetze“, die das "geschichtliche Leben“ in seinem "Kreislauf zwischen menschlichem Sein und menschlicher Selbstdeutung " beherrschen. Es verläuft dieses geschichtliche Leben in den Polaritäten von Partikularismus und Universalismus, Versinnlichung und Vergeistigung, Autonomie und Arbeitsteilung. Rothacker macht zum Ziel seiner Kulturanthropologie „eine vollständige Systematik aller polaren Dimensionen, in denen menschliches Streben überhaupt sich bislang historisch bewegt hat, und unter noch umfassenderem Gesichtspunkt überhaupt bewegen kann. ${ }^{\text {“36 }}$

Wenn Walter Haugs drei Dilemmata nicht nur rhetorischer Natur sind, dann entsprechen sie diesem Denken, einem in ahistorische Polaritäten auflösbaren historischen Denken, dessen Horizont die dem historisch Wechselhaften abgerungenen Konstanten sind. Waiter Haugs „Konstanz des Dichterischen“ wäre in der Tat keine ganz ungeeignete Kandidatin für den Dilthey-Rothackerschen Typ von „Kulturanthropologie“. Und sein Versuch, die literaturwissenschaftliche "Geschichte“ vor den kulturwissenschaftlichen Zudringlichkeiten in Sicherheit zu bringen, führte diese Literaturwissenschaft nur desto tiefer in die kulturwissenschaftliche Debatte hinein. Denn die Annahme von „Konstanten“ im oder gegen den Prozeß des „Historischen“ ist eines der Kernprobleme der

${ }^{35} \mathrm{Vgl}$. Odo Marquard, „Leben und leben lassen. Anthropologie und Hermeneutik bei Dilthey", Dilthey Jabrbuch 2 (1984), 128-139.

${ }^{36}$ Christian Grawe, „Kulturanthropologie“, in: Joachim Ritter, Karlfried Gründer (Hrsg.), Historisches Wörterbuch der Philosophie, IV: J-K, Darmstadt 1976, $1326 \mathrm{f}$. 
aktuellen Diskussion um Historische Anthropologie, um die historischen Semantiken von „Philosophischer Anthropologie“, „Kulturanthropologie“ und "cultural anthropology“. Dabei geht es nicht um das kasuistische Argument, daß jemand, der gegen Kulturwissenschaft argumentiert, sich notwendigerweise an der kulturwissenschaftlichen Debatte beteiligt. Nein, Walter Haugs „Konstanten" sind Teil eines kulturwissenschaftlichen Modells, das seit Erich Rothacker den Namen der „Kulturanthropologie“ trägt.

Ob sein Galopp aus der Geschichte auf dem Rücken der „Konstanten“ Walter Haug so ganz berechtigt, den Verlust der Geschichte im New Historicism zu beklagen, stehe dahin. Sicher ist, daß Stephen Greenblatt nicht die, sondern allenfalls eine Geschichte verläßt. Ein Vorzug einer kulturwissenschaftlichen Relativierung des Eingeübten ist nicht zuletzt die Wiederentdeckung der Tatsache, daß es verschiedene Möglichkeiten gibt, die „Geschichte" zu konstruieren und daß die Abkehr vom Dilthey-Rothackerschen Typ der Geisteswissenschaft, Geistesgeschichte und Kulturanthropologie noch keineswegs die Preisgabe von Geschichte und Wahrheit an "postmoderne Unverbindlichkeit" bedeutet.

Walter Haug sieht ganz richtig, daß es bei Greenblatt um „Heterogenität“, ums „Kontingente“, um „Widersprüchlichkeiten“ und um „Brüche“ geht. Daß dahinter ein Konzept von "Geschichte" steht, ist ihm vielleicht entgangen, weil er Greenblatt zu eng an Geertz' „dichte Beschreibung“ bindet und übersieht, daß mindestens so wichtig wie Geertz das Foucaultsche Vorbild für Greenblatt ist. Die von Walter Haug erwähnten Machtanalysen sind an Foucault geschult. Um es sehr vereinfachend zu sagen: Foucaults Archäologie des Wissens behauptet, daß eine auf die Prinzipien von „Diskontinuität, Bruch, Schwelle, Grenze, Serie ${ }^{\text {“ }}{ }^{37}$ aufgebaute Methodologie alles das ans Licht der Erkenntnis holt, was die auf die Kohärenzfiguren Autor, Werk, Einfluß, Tradition, Entwicklung, Mentalität oder Geist gegründete Geschichtsschreibung ausgeblendet hat. Mit Blick auf die Wissenschaftsgeschichte kann man wiederum sehr vereinfachend hinzufügen, daß es eine gleich große aber nicht austauschbare Erkenntnisleistung der auf Kohärenz und der auf Nicht-Kohärenz gegründeten Verfahren gibt. Ein typisches Kohärenz-Verfahren ist z.B. die historistische Einflußforschung. Als „Einfluß“ gilt im Idealfall nur, was ohne Lücke und in positiv nachweisbaren Textspuren sichtbar zusammenhängt. Ein ebenso typisches Verfahren der Nicht-Kohärenz hat Stephen Greenblatt für sich in Anspruch genommen und in einem Clifford Geertz gewidmeten Sonderheft seiner Zeitschrift Representations auf den Punkt gebracht. Greenblatt stellt dort dar, was er bei Geertz gelernt hat und kommt zu einem Schluß, der Walter Haug wie Musik in den Ohren klingen müßte.

"For the interest was never to collapse anthropology and literary criticism

${ }^{37}$ Michel Foucault, Archäologie des Wissens, Frankfurt a.M. 1973, 33. 
into each other but to draw upon their particular strengths, strengths, that depended at least as much upon the differences between their characteristic texts as upon their surprising similarities." Vor allem aus der Differenz des Literarischen und Nicht-Literarischen macht Greenblatt ihrer beider Konstellation produktiv. „That both the literary work and the anthropological (or historical) anecdote are texts, that both are fictions in the sense of things made, that both are shaped by the imagination and by the available resources of narration and description helped make it possible to conjoin them, but their ineradicable differences - the fact that neither is purpose-built for the other, that they make sharply different claims upon the actual, that they are incommensurable and virtually impossible to foveate simultaneously - made the conjunction powerful and compelling. " ${ }^{38}$

Greenblatt schwächt die ontologische Differenz von Nicht-Fiktionalem und Fiktionalem ab zugunsten der chronologischen und genealogischen Differenz. Ein Geisterseher-Bericht von 1668 und die Geisterszenen im Hamlet haben ein gemeinsames Thema und sind doch durch die genannten Differenzen bis zur Beziehungslosigkeit getrennt: Unähnlichkeit des Ähnlichen, discors concordia. Erst das Herausarbeiten der kulturellen Faktoren dieser chronologischen und genealogischen Differenzen erschließt den Erkenntniswert der thematischen Ähnlichkeit. Als Erkenntnismethode hat diese Verähnlichung des Unähnlichen eine lange Tradition. $\mathrm{Zu}$ beginnen hätte ihre Geschichte beim Xenikón des Aristoteles. „Die ,fremde‘ Sprache und die ,unbekannten“ Gründe“ wurden zur Quelle für „ästhetische und kognitive Verfahren“"39. Für die ästhetischen Verfahren wäre zum Beispiel die concettistische Poetik des Wunderbaren anzuführen. Ihre Wirkung zeigt Greenblatt in einem anderen Aufsatz an Kolumbus' Rhetorik der „Verwunderung“, die aus der staunenden Begegnung mit dem Fremden eine Strategie seiner Überwältigung und Aneignung macht. ${ }^{40}$ Greenblatt selbst praktiziert den rhetorischen concetto mit seiner Verbindung von Hamlet-Szenen und jüngerem Geister-Bericht, er begründet seine Konfigurationen des „Deplacierten“, die Verunähnlichungen des Ähnlichen aber nicht mit der concettistischen Rhetorik, sondern mit den „Verfremdungs"-Verfahren der Formalisten ${ }^{41}$ ihrem methodischen Aufbrechen des Automatisierten und Konventionalisierten in der Differenz. So wurde bekanntlich Roman Jakobsons dichterische Erfahrung der poetischen Differenz zu einem Ausgangspunkt

${ }^{38}$ Stephen Greenblatt, "The Touch of the Real“, Representations 59 (1997) (Special issue, The Fate of ,Culture ${ }^{\epsilon}$ Geertz and Beyond, ed. by Sherry B. Ortner), 14-29; 20, 22.

39 Renate Lachmann, Die Zerstörung der schönen Rede. Rhetorische Tradition und Konzepte des Poetischen, München 1994, 101.

${ }^{40}$ Stephen Greenblatt, Wunderbare Besitztümer. Die Erfindung des Fremden: Reisende und Entdecker, 3. Kapitel: „Wunderbare Besitztümer“, Berlin 1998, 87-132.

41 Stephen Greenblatt (Anm. 38), 26. 
für seine linguistischen Analysen der Alltagssprache. Der concetto ist unter der Bezeichnung "phantastischer Gattungsbegriff" durch Giambattista Vico in die neuzeitliche Wissenschaft eingeführt worden. Vico charakterisierte mit dem "phantastischen Gattungsbegriff“ das mythische Denken, paßte diesem Gegenstand auch seine eigene rhetorisch fundierte Methode an, um eine gegen den cartesianischen Methodenbegriff gerichtete historische Wissenschaft zu begründen. Über Empirismus und Positivismus kam der cartesianische Methodenbegriff zur Herrschaft in den historischen Wissenschaften. Gegen seine Kohärenz- und Systemzwänge gab es immer alternative Konstruktionen des Wissens und Erkennens. An den romantischen Fragmentarismus und Encyclopädismus wäre zu erinnern. In der neueren Wissenschaft hat vor allem Georg Simmel die systemlose Verknüpfung des Heterogenen virtuos als wissenschaftliches Verfahren praktiziert. Adorno hat, nicht zuletzt auf Simmel gemünzt, diese Differenz-Methode wieder direkt in Stellung gegen Descartes' Discours de la méthode gebracht. Der cartesianische Methodenbegriff hilft, die Fiktion einer logisch aufgebauten Welt aufzubauen, nicht aber die kontingente Geschichte der Kultur zu verstehen. „Kontinuierliche Darstellung widerspräche einer antagonistischen Sache, solange sie nicht die Kontinuität zugleich als Diskontinuität bestimmte. “42

Walter Haug wird seine Abneigung gegen Adorno bestätigt finden. Gleichwohl gehört Adornos Essay als Form zu den kanonischen Texten, die den mit Vico begründeten Wissenstyp des „Nichtidentischen“ beschreiben. Der große Nachteil von Adornos Essay als Form ist nur, daß er den Verächtern dieses Methodenideals einen Vorwand liefert, es als „essayistisch“ im Sinne von schlampigem Feuilletonismus zu diskreditieren oder ihm, ein wenig freundlicher, ,anekdotische Anschaulichkeit“ und „postmoderne Unverbindlichkeit“ zu bescheinigen. Daß ein schlechter Essay nicht schlechter ist als eine schlechte Abhandlung, bleibt dabei ebenso außer Betracht, wie die Tatsache, daß es historische Felder gibt, die sich wissenschaftlich besser rekonstruieren lassen, wenn man sie nicht in positiven linearen Zusammenhängen vermißt, sondern wenn man die unsichtbaren Vernetzungen ihrer Disparatheiten als möglich aufzeigt.

Man kann wie Walter Haug sagen, das Ergebnis des New Historicism sei „keine Geschichte“, sondern eine „Essay-Folge“. Wenn das als vernichtende Feststellung gemeint sein sollte, dann ist die Eleganz zu bewundern, mit der in einem Halbsatz eine ganze Wissenschaftstradition beiseite gewischt wird, eine Wissenschaftstradition des „Erkennens durch Verfremden“, die im GeertzGreenblattschen Aufgreifen des Simmelschen Essay-Begriffs und in der Foucault-Greenblattschen Praxis des Diskontinuierlichen zu einer aktuellen Ver-

42 Theodor W. Adorno, Der Essay als Form, in: ders., Noten zur Literatur I, Frankfurt a.M. $1969,9-49 ; 34 \mathrm{f}$. 
sion gefunden hat. Es wäre eine eigene Überlegung wert, ob eine nicht auf erkennende Aneignung des Fremden, sondern auf erkennende Bewahrung des Fremden gerichtete, eine kulturwissenschaftliche Hermeneutik des Fremden also, naturgemäß größere Affinitäten zur kognitiven Leistung des aristotelischen Xenikón hat, als eine literaturwissenschaftliche Hermeneutik, die sich darauf beschränkt, im Kanon des Eigenen die Schatten des Fremdwerdens aufzuhellen.

Sicher hat Walter Haug recht, wenn er diese literaturwissenschaftliche Hermeneutik als "dilemmatische“ charakterisiert. Statt die Literaturwissenschaft aber auf drei Dilemmata zu gründen, würde ich ihr vorschlagen, sich auf drei Herausforderungen einzulassen. Sie könnte sich den materiellen und strukturellen Veränderungen unserer Kultur stellen, ohne sich vorschnell mit dem "Konstanten“ und „Identischen “ zu immunisieren. Sie könnte die doppelte Moral von pluralistischer Praxis und monistischer Selbstdefinition durch Konzeptualisierungen des pluralistischen Denkens ablösen. Sie könnte schließlich in vielen ihrer Begriffe und Kategorien durch deren Reformulierung im kulturwissenschaftlichen Kontext, neue und spannende, in ausgezeichneter Weise die Literatur betreffende Fragestellungen entdecken. Literatur ist für die Literaturbegeisterten und die Literaturwissenschaftler das Höchste. Die Berechtigung zu dieser Einstellung hat kulturelle Gründe, aus deren Berücksichtigung die Begeisterten wissenschaftlichen Nutzen ziehen können. 